\title{
Resolving Nanostructured Materials Down to the Single-atom Limit
}

Xiangbin Cai ${ }^{1}$, Antonio Tricoli ${ }^{2}$, Hongyang Liu $^{3}$, Yuan Cai ${ }^{1}$, Christian Dwyer ${ }^{4}$, Ning Wang ${ }^{1}$ and Ye Zhu ${ }^{5}$

${ }^{1}$ Hong Kong University of Science and Technology, Hong Kong, Hong Kong, ${ }^{2}$ Australian National University, Canberra, Australian Capital Territory, Australia, ${ }^{3}$ Chinese Academy of Sciences, Shenyang, Liaoning, China (People's Republic), ${ }^{4}$ Arizona State University, Tempe, Arizona, United States, ${ }^{5}$ Hong Kong Polytechnic University, Hong Kong, Hong Kong

Determining the structural variation and the chemical inhomogeneity, ideally at atomic resolution, is critical for the understanding of nanostructured materials' functionality. However, the limited volume of parent matrix and the even smaller scale of local structural or chemical fluctuations impose great challenge onto the effective characterization, because conventional structure-analysis techniques can only provide information averaged over the whole or part of sample, overlooking the ordering spatially localized within few nanometers. ${ }^{[1]}$ The maturation of aberration-corrected electron microscopy instrumentation in the past decades has enabled the extraction and quantification of weak scattering signals from individual atoms in nanostructured materials. Here we present how this powerful tool can reveal the dopant nanophases in nanoparticles and the composition-modulated dispersion behaviors of bimetallic catalysts down to the single-atom limit. Doping has served as a popular strategy to tailor chemical and physical properties of today's materials, whose effects become increasingly important when the host dimension scales down. Rational synthesis of doped nanocrystals thus requires precise knowledge on the atomic structure and electronic configuration of each dopant-atom introduced. Using the 3 at\% Ce-doped $\mathrm{Mn}_{3} \mathrm{O}_{4}$ nanoparticles of $<10 \mathrm{~nm}$ size as a model system, we demonstrated atomic-scale oxidation-state mapping and quantitative dopant-atom counting by the electron energy-loss spectroscopy in an aberration-corrected scanning transmission electron microscope (STEM-EELS). ${ }^{[2]}$ In Figure 1(a), the oxidation states of Ce dopant-atoms in different coordination environments were identified, i.e. the $\mathrm{Ce}^{4+}$ single ions and clusters embedded inside the charge-ordered $\mathrm{Mn}_{3} \mathrm{O}_{4}$ matrix, and the partially reduced $\mathrm{Ce}^{3+\mathrm{x}}$ atomic layers on certain crystal facets. Moreover, the electron-scattering simulations and the refined detecting set-up allow us to quantify the dopant-atom number in each atomic column and derive a 3D dopant structure of the few-unit-cell $\mathrm{CeO}_{2}$ nanocluster beyond a 2D projection, as shown in Figure 1(b). It's also surprising to find that STEM-EELS can pinpoint the dopant-atom position more accurately than the annual dark field (ADF) imaging. When the $\mathrm{Ce}^{4+}$ ions were displaced away from the substituted $\mathrm{Mn}^{3+}$ column, the simultaneous ADF failed to reflect the correct dopant position while STEM-EELS worked well, which indicates that the Ce intercalation may not be so coherent as ADF images look. Corroborated by simulations, STEM-EELS is proved to be more sensitive to small displacement of dopant-atoms and suffers less from channeling artefacts than the ADF imaging. The Pt-Sn catalysts supported on nanocarbons have shown advantages of high thermal stability, enormously enhanced reaction activity and selectivity over traditional Pt products for the direct dehydrogenation of $n$-butane, but how the bimetallic structure varies with the composition remains elusive. ${ }^{[3]}$ In Figure 2, we demonstrated that the addition and coordination of $\mathrm{Sn}$ atoms act as an effective dispersion agent to Pt species, resulting in the prominent tunability of the dispersed metal sizes, the active-center structure and thus the catalytic performance of Pt$\mathrm{Sn}$ catalysts. With increasing Sn concentration, the bimetallic nanostructure evolves gradually from $\mathrm{Pt}$ crystals $(\sim 5 \mathrm{~nm})$, Pt-Sn core-shell nanoparticles $(\sim 3 \mathrm{~nm})$, fully exposed Pt-Sn single atomic layers $(\sim 1$ $\mathrm{nm})$, Sn-rich bimetallic nanoclusters $(\sim 1.5 \mathrm{~nm})$, finally to segregated $\mathrm{Sn}$ and Pt clusters $(\sim 2 \mathrm{~nm})$. Figure 2(b) shows how quantitatively $\mathrm{ADF}$ can uncover the atomic arrangement and chemical identity of 
supported Pt-Sn catalysts with single-atom sensitivity. In summary, the single-atom-resolved capabilities provided by the modern aberration-corrected electron microscopy and spectroscopy are widely applicable to other nanostructured materials, such as the twisted bilayers of transition metal dichalcogenides, which exhibit reconstructed moiré domains regulated by the marginal twist angles. These quantitative techniques may also be extended to facilitate the in-situ study of materials' functionality under realistic conditions. ${ }^{[4]}$

(a)
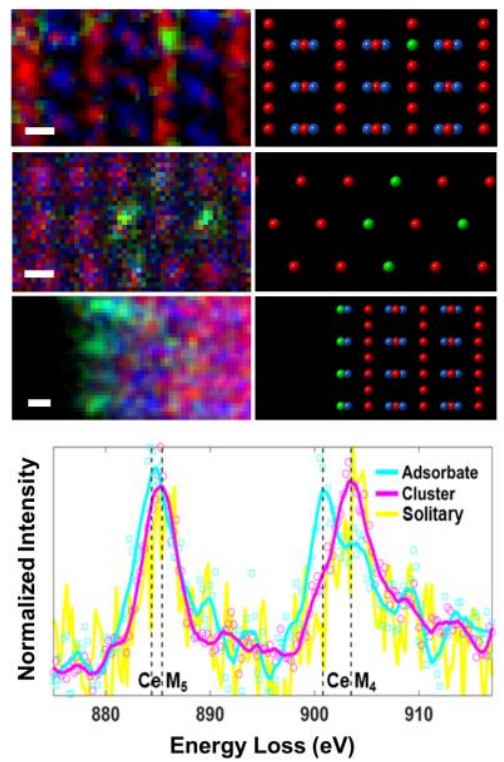

(b)
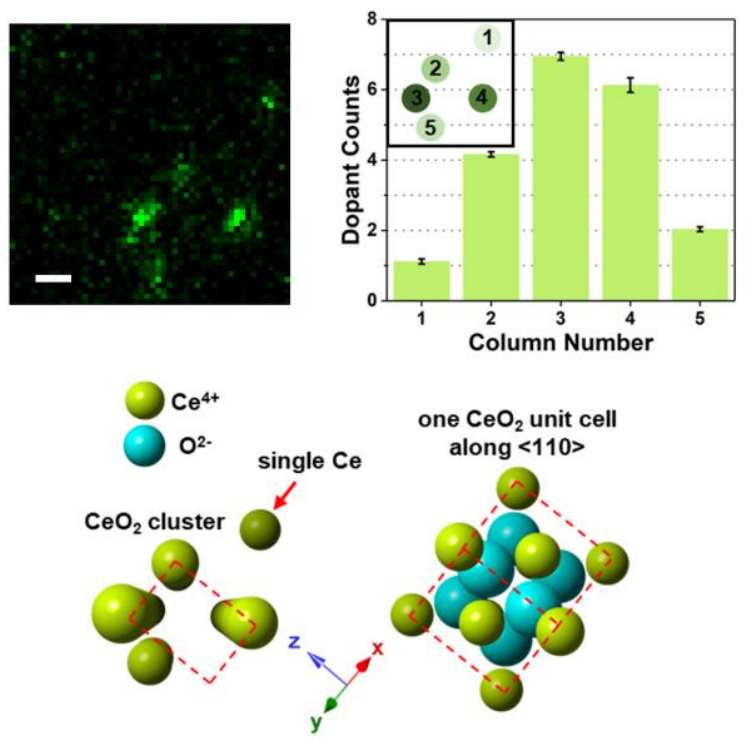

Figure 1. (a) STEM-EELS mapping of various dopant nanophases, including the Ce4+ single ions and clusters inside $\mathrm{Mn3O} 4$ nanoparticles and the $\mathrm{Ce} 3+\mathrm{x}$ atomic layers on the Mn3O4 \{101\} facets. Red, blue and green colors represent $\mathrm{Mn} 3+, \mathrm{Mn} 2+$ and $\mathrm{Ce} 4 / 3+$, respectively. (b) Quantitative dopant-atom counting revealing a $\mathrm{CeO} 2$ nanocluster. The scale bars are $2 \AA$. Reprinted and adapted with permission from reference [2]. Copyright (2019) American Chemical Society.

(a)
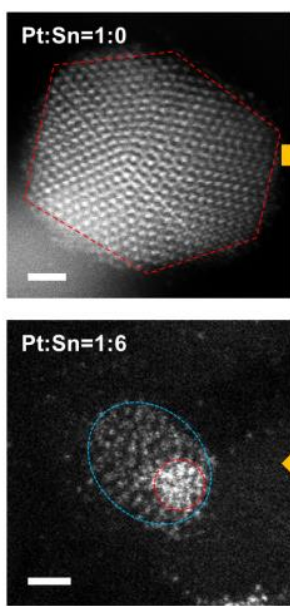
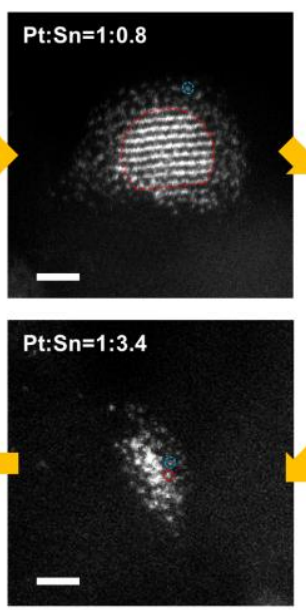

(b)
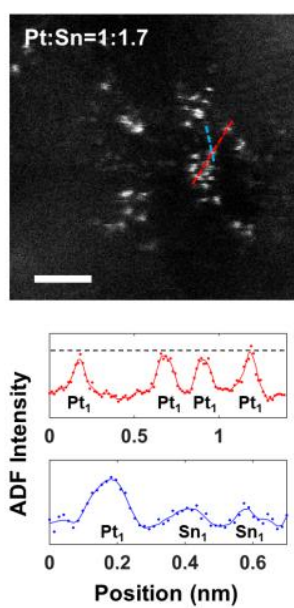

Figure 2. (a) Structural evolution of the catalytically active centers in Pt-Sn catalysts, which is modulated by the Sn concentration. (b) Fully exposed Pt-Sn single atomic layers with line profiles showing the singleatom sensitivity of ADF. The scale bars are $1 \mathrm{~nm}$. 


\section{References}

1. Billinge and I. Levin, Science 316, 561-565 (2007).

2. Cai et al., Chemistry of Materials 31, 5769-5777 (2019).

3. Zhang et al., ACS Catalysis 9, 5998-6005 (2019).

4. We acknowledge the financial assistance from the Research Grants Council of Hong Kong (Project Nos. 25301617 and N_HKUST624/19) and the facility support of JEOL JEM ARM 200F at the MCPF of HKUST. 